\title{
IDEOLOGIA E POESIA DI EUGENIO MONTALE DA LA BUFERA E ALTRO A SATURA
}

Carmelo Distante

\begin{abstract}
Abbiamo scelto come oggetto di questo studio su Eugenio Montale il tema l'ideologia e la poesia da La bufera e altro a Satura, perché a noi pare che è in questi due libri che il poeta genevose raggiunga la piú compiuta e matura espressione della sua umanità .

Intendiamoci, con questo non vogliamo dire affato che in queste due raccolte è dato trovare una concentrazione poetica maggiore che nelle altre, di prima e di dopo, bensí solamente che ne La bufera e altro e in Satura viene a maturazione e, per cosí dire, si svela tutta la complessa umanità del poeta. Tanto è vero che possiamo aggiungere che le altre raccolte, da Ossi di seppia a Le Occasioni, dal Diario del '71 e del 72 al Quaderno di quattro anni, le prime due antecedenti a La bufera e le seconde posteriori a Satura, si possono considerare sempre sí come libri di grandissima poesia che trova in se stessa la sua ragione di essere, ma pure come libri che se letti da soli non ci danno tutta la misura di Montale uomo e poeta, come si rivela ne La bufera e in Satura. La verità è, infatti, che tra La bufera e Satura quasi tutti i nodi della precedente poesia montaliana vengono allo scioglimento e quella che segue non indica strade nuove, ma ripescorre, sia pure con canto sempre alto e sostenuto, vie già note a chi conosce le altre raccolte del nostro poeta.
\end{abstract}

$\mathrm{E}$ bene ora, prima di procedere, fare qualche accenno, e sia pure, in modo estremamente sintetico, alla posizione morale e ideale assunta da Montale sin dall'apparire del suo primo libro di poesie, Ossi di seppia, e poi nel secondo, Le occasioni, e metterne subito in evidenza l'originalità e la profondità che lo hanno portato ad essere, su scala mondiale, il poeta forse piú grande del nostro secolo, il simbolo stesso di tanta parte della coscienza dell'uomo del Novecento impegnato a scoprire il senso e il significato del suo essere al mondo. E a questo proposito non possiamo fare a meno di ricordare, proprio per meglio capire l'originalità e la profondità della posizione montaliana, le principali idee-forza o, se si vuole, i miti che hanno sorretto per lungo tempo l'uomo moderno. 
E noto che il Novecento ha ereditato dall'Ottocento, che a sua volta l'aveva ereditato dal Settecento, l'idea e il concetto di progresso (e non importa qui mettere in luce tutte le differenze che pure ci sono, e non di poco conto, tra il Settecento illuminista, l'Ottocento romantico e il Novecento aperto e proteso al massimo a tutte le avventure intelletuali e spirituali) L'idea di progresso è una costante che ha informato il mondo moderno. Possiamo aggiungere solamente che nel Novecento quest'idea ha trovato il suo epilogo e insieme la sua crisi, crisi che attualmente stiamo vivendo e della quale la poesia di Montale è forse la testimonianza piú alta e piú complessa.

L'idea di progresso ha come base una concenzione ottimistica, se non della vita dell'individuo, certamente dello sviluppo e del divenire storico dell'umanità. Coerentemente con questa concezione, tutta la storia è vista come una serie di gradi e di passaggi che portano l'uomo da uno stadio piú basso ad uno stadio piú alto di civiltà e cosí da uno stadio di minore ad uno stadio di maggiore libertà ad infinitum. Sicché si è salutata ogni scoperta filosofica e scientifica, allo stesso modo di ogni realizzazione poetica e artistica, effettuata nel corso dei secoli, come una liberazione anche morale dell'uomo. $\mathrm{Va}$ da sè che questo cammino dell'umanità non è visto come progrediente in modo rettilineo, bensí mediante passaggi dialettici, vale a dire mediante l'assorbimento del negativo nel positivo e il superamento di quello da parte di questo. Insomma, l'uomo si può anche momentaneamente fermare o anche retrocedere, ma poi, alla fine, non può che trovarsi piú avanti del punto di partenza. La sua marcia allora non può essere che una marcia progrediente, sia pure mediante un cammino che può conoscere fermate e bensí momentanee retrocessioni.

E vero che occorrerebbe ora fare delle distinzioni per articolare in senso storico-critico il discorso su questa idea di progresso che tanto in profondità ha informato la coscienza del mondo moderno. Non si può, infatti, non tener conto della grande differenza di posizione nei confronti delle sorti e del destino degli uomini che esiste, per esempio, tra un poeta pessimista e materialista come Giacomo Leopardi, il piú grande poeta italiano dell'Ottocento e uno dei massimi di tutto il mondo, e gli altri spiritualisti o cattolici romantici, compreso lo stesso Manzoni, cosí come non si può non tener conto della profonda differenza che c'è nei confronti dell'idea di progresso e del modo in cui questo si attua, tra un filosofo conservatore come Hegel e un pensatore rivoluzionario come Marx; o della grande differenza che esiste, per prendere in considerazioni due altri grandi italiani del nostro secolo, tra il pensiero di Croce e quello di Gramsei, circa il modo in cui procede e si attua lo sviluppo della storia e quindi 
circa il modo in cui si snoda la marcia degli uomini dalla necessità verso la libertá. Ma sta di fatto che, al di lá differenze ideali e delle posizioni pratiche assunte da questo o da quel movimento di pensiero, si può tranquillamente affermare che nulla si è realizzato nel mondo, almeno a cominciare dalla revoluzione francesa in poi, se non in nome del progresso e della liberazione ad infinitum dell uomo. Tutte le lotte politiche e sociale, tutte le rivoluzioni, tutte le guerre d'indipendenza nazionale, e anche le stesse guerre imperialistiche intraprese per il dominio coloniale o per la conquista del mercati, hanno avuto come giustificazione (e non sembri un'ironia) l'idea di progresso e di liberazione dell' uomo. $E$ ovviamente anche le guerre e le lotte che i popoli colonizzati hanno dovuto fare per liberarsi dal dominio coloniale. Sicché non sempre è facile tracciare una linea di delimitazione netta nella storia del mondo moderno e contemporaneo tra movimenti rivoluzionari realmente progressisti e movimenti rivoluzionari soltanto a parole, ma in raltà reazionari ed oppressivi. $\mathrm{E}$ questo si può dire sia per quanto riguarda i movimenti di struttura, vale a dire quelli politici ed economici im senso stretto, che per quanto riguarda $i$ movimenti sovrastrutturali, cioè ideali, culturali ed artistici che dei primi sono sempre la proiezione, si voglia o non si voglia.

Orbene, ritornando ora a Montale, vediamo qual è la posizione che egli assume sin dagli esordi di fronte a questo problema, vale a dire di fronte al problema del destino e dell'essere dell'uomo moderno nel mondo, collocandosi al centro di un dibattito ideale e morale che il nostro secolo, come abbiamo detto sopra, ha ereditato dal Settecento illuminista e dall' Ottocento prima romantico, poi positivista e infine decadente. Non ci soffermeremo a lungo su questo Montale, vale a dire su Montale autore di Ossi di seppia e de Le occasioni, perché il nostro discorso vuole avere come oggetto specialmente il Montale de La bufera e di Satura, cioè di un tempo che si colloca tra il 1940, che é l'anno dell'inizio della seconda guerra mondiale, il 1945, che è l'anno che segna la fine della stessa guerra, e gli anni che seguono alla fine di questa, sino al 1970, che è l'anno, appunto, con cui si chiudono le poesie di Satura. Tuttavia occorre fare qualche accenno al Montale degli anni 1920-1940, proprio per capire meglio la posizione ideologica e poetica del Montale degli anni tra il 1940 e il 1970.

Ossi di seppia, come è noto, è il primo libro poetico di Montale e fu composto, come l'autore stesso ci fa sapere in una nota, tra il 1920 e il 1927, se si esclude Meriggiare pallido, che risale al 1916. Sono gli anni dunque che seguono alla prima guerra mondiale e sono gli anni cruciali in cui il fascismo s'impadronisce del potere in Italia. Le scelte da fare sembrano chiare: o si è di qua o si è di là, o si è fascisti o si è antifascisti. Croce, come la piú valida cultura italiana, passa 
all'opposizione; Gramsci viene imprigionato e dal fondo di una cella forgierà le armi per la riscossa del proletariato. Intanto però, stando in galera, s'infiacchisce nella salute e poi perde addirittura la vita. I fascisti da parte loro credono di avere salvato la nazione dal marasma e di averla avviata verso un avvenire di grandezza illimitata. Imsomma, mentre i fascisti da una parte credono che col loro avvento al potere tutti i mali della nazione saranno avviati a soluzione e che la stessa oliventerà grande e potente e gl'italiani rispettati e temuti, gli antifascisti dall' altra non perdono la speranza che un giorno l'Italia ritornerà libera $\mathrm{e}$ il popolo riprenderà a vivere con dignità $\mathrm{e}$ non oppresso. Cosí sia i fascisti che gli antifascisti si fanno guidare nella loro azione e nelle loro scelte da una fede quanto si vuole opposta, anzi contrapposta, ma pur sempre ottimistica. Non ci scordiamo che sarà lo stesso Gramsci che teorizzerà in carcere che l'uomo nella vita e nella storia è necessario che si faccia guidare dal pessimismo della ragione e dall'ottimismo della volontà. Pensava, dunque, che nonostante tutto gli uomini non debbano mai rassegnarsi al male, all'infelicità e alla sconfitta, anzi li esortava ad impegnarsi con tutte le forze della volontà a cercare di costruirsi una vita e una storia che fossero coronate dal successo, sebbene con la ragione dovessero sempre sapere che l'esito di ogni impresa umana non è mai scontata in partenza .

Come Montale, intanto, si poneva di fronte alle cose? Quale posizione cioè assumeva? Come, in altri termini, gli pareva il mondo e la sorte dell'uomo nello stesso? Niente ci può aiutare di piú a rispondere a queste domande che richiamare alla nostra attenzione la prima lirica con cui si aprono i veri e propri Ossi di seppia. E un componimento celebre che tutti conoscono. Si tratta di quello che comincia col verso Non chiederci la parola che squadri da ogni lato. E evidente che in questa poesia il poeta assumeva una posizione fortemente negativa non solo di fronte allideologia fascista, sebbene fosse a questa che particolarmente s'indirizzava il suo no, ma di fronte a tutte le ideologie che in qualche modo volevano che si rispondesse facilmente e chiaramente al problema dell'essere e del destino dell'uomo nel mondo. In raltà Montale esprimeva in termini poetici altissimi la coscienza della grande difficoltà che l'uomo contemporaneo trova a pronunciarsi sulla ragione e sul senso del suo essere sulla terra. Il mondo gli appariva non decadentisticamente come un mistero che soltanto il poeta può svelare, ma come un problema difficilissimo la cui soluzione non è alla portata di mano di nessuno, né degli uomini comuni, né degli scienziati, né dei poeti. Perciò vana e piú che vana, stolta, gli pareva la pretesa di tutti coloro che vogliono e credono che la realtà sia qualche cosa di chiaro e di semplice. E il poeta chiudeva questa grande lirica con la famosissima quartina, che si può considerare come il suo testamento poetico e ideale degli anni in cui compose Ossi di seppia: 
Non domandarci la formula che mondi possa apriti, sí qualche storta sillaba e secca come un ramo.

Codesto solo oggi possiano dirti, ciò che non siamo, ciò che non vogliamo.

E chiaro che qui Montale respingeva tutte le "bacature" culturali e ideologiche ottocentesche e novecentesche: respingeva la pretesa positivistica di spiegare il mondo con la scienza, quella decadente di scoprire e impossessarsi dell'assoluto con l'arte, cosí come respingeva l'attivismo ottimistico futurista e delle altre avanguardie e nello stesso tempo annunciava chiaramente che il compito dell'uomo contemporaneo non è quello di emettere facili sentenze sul mondo e sulla storia o di abbracciare delle filosofie, come quelle idealistiche o attivistiche, che sembrano rispondere con chiarezza e completezza a tutto, ma che in realtà non rispondono a niente, bensí di rifiutarsi di dare facili giudizi su cose che non si sa che cosa siano né dove portino, tanto che ammoniva: codesto solo oggi possiamo dirti, / ciò che non siamo, ciò che non vogliamo. E si noti che sottolineava le due negazioni, proprio per mettere in rilievo che l'unica cosa di cui l'uomo contemporaneo può dirsi sicuro è del fatto che non sa chi sia né che cosa veramente voglia.

Un altro componimento molto importante per capire l'atteggiamento del poeta di fronte alla vita al tempo degli Ossi di seppia, è quello che comincia col verso Spesso il male di vivere ho incontrato. Si tratta anche in questo caso di un componimento famoso. Perciò non ci pare il caso di soffermarci a lungo su di esso. Diremo soltanto che qui l'autore manifestava ancora una volta chiaramente la sua visione della vita non programmaticamente ottimistica, anzi tutto il contrario. Ma è la seconda quartina che c'interessa in special modo. Cantava il poeta:

\author{
Bene non seppi, fuor del prodigio \\ che schiude la divina Indifferenza: \\ era la statua nella sonnolenza \\ del meriggio, e la nuvola, e il falco alto levato.
}

È chiara l'indisponibilità di Montale per gli entusiasmi e gl'incantesimi ideologici. Il bene non consiste nell'adesione, piú o meno motivata, alle ideologie, ma se mai nell'indifferenza di fronte alle stesse. Non è, in altri termini dalla partecipazione emozionale a qualche credo ideologico che ci possa derivare il bene morale, bensí al contrario dal distacco con cui sappiamo partecipare alle passioni della vita. $\mathrm{E}$ a questo proposito molto significativo ci pare anche quanto scriveva in Stile e tradizione, un articolo pubblicato su "Il Baretti", anno I n. I, il 15 gennaio del 1925, poi ristampato con "piccoli ritocchi" 
in Auto da fè, dove accennando alla debolezza stilistica di scrittori e poeti come Carducci, Pascoli e D'Annunzio, scriveva: "Lo stile, il famoso stile totale che non ci hanno dato i poeti dell'ultima illustre triade, malati di furori giacobini, superomismo, messianismo ed altre bacature, ci potrà forse venire da disincantati savi ed avveduti, coscienti dei limiti e amanti in umiltà dell'arte loro piú che del rifare la gente. In tempi che sembrano contrassegnati dall'immediata utilizzazione della cultura, del polemismo e delle diatribe, la salute è forse nel lavoro inutile e inosservato: lo stile ci verrà dal buon costume" Dove è evidente che l'autore prendeva posizione contro chi confondeva la poesia con l'ideologia e faceva chiaramente intendere che non ci poteva essere salute innanzi tutto per i poeti, ma poi anche per gli uomini in generale, se non liberandosi dalla pretesa che per risolvere i problemi che travagliano l'uomo, basta farsi adepti di qualche ideologia e proclamare questa come la salvatrice del mondo. La vita invece gli pareva che andava vissuta come pena chiusa e come realtà difficilmente decifrabile. Il male e il dolore inoltre li vedeva come elementi che accompagnano ad ogni passo il fenomeno fisico e metafisico dell'esistenza. Si pensi ancora alla lirica Spesso il male di vivere ho incontrato o a quella che comincia coi versi Mia vita, a te non chiedo lineamenti / fissi, volti plausibili o possessi. E si può dire che tutto il libro di Ossi di seppia è percorso da un profondo scandaglio circa la chiusa e difficile essenza del mondo e della vita. E questa è l'importanza del segno storico-artistico, oltre che morale, di quel grande libro. E chiaro, infatti, che Montale con Ossi di seppia, in un tempo in cui la retorica fascista sembrava trionfare soddisfatta di sé, indicava agli uomini il volto estremamente problematico, oltre che duro e nascosto delle cose. Non si trattava però di un volto disperato, e su questo punto occorre richiamare con forza l'attenzione, nonostante che pure le cose gli apparivano amare, difficili, incerte e spesso enigmatiche. La speranza, insomma, spuntava dall'interno della disperazione. E per questo aspetto di speranza, per cosí dire, disperata, di ansia cioè che tende ad abbandonare le viscere oscure della terra per proiettarsi verso gli "azzurri specchianti del cielo", si veda un componimento come Portami il girasole.

Ma è ne Le occasioni, composte tra il 1929 e il 1939, nel decennio cioè che vide l'apparente quanto tronfio rafforzamento del fascismo in Italia, l'ascesa del nazismo in Germania, la conquista dell'Etiopia da parte della stessa Italia fascista e il soffocamento nel sangue della libertà spagnola da parte del franchismo, appoggiato dalle forze naziste e fasciste, nel decennio, in altri termini, che preparò all'Europa e al mondo la tragedia della seconda guerra mondiale, che Montale consolida la sua visione del mondo oscura e chiusa, eppure solcata di tanto in tanto, da un qualche raggio improvviso di luce che sembra squarciare, quasi in modo intermittente e magari 
quando meno ce se lo aspetta, le tenebre che incombono sulla terra, onde dare all'uomo che vive sulla crosta della stessa la coscienza che la vita è sí problematica ed oscura, difficile e dura, ma non tale che di tanto in tanto chi la viva non possa scorgere in essa una qualche luce che forse, chi lo sa? lo può anche salvare. Non c'è niente di programmatico e di sicuro, ma tutto è possibile.

Il poeta, come è noto, stampava, in limine a Le occasioni, una poesia come Il balcone, che faceva parte dei Mottetti, "per il suo valore di dedica" Ebbene, è proprio nel Il balcone che possiamo trovare la chiave che ci può permettere di aprire il chiuso significato di tutta la poesia del libro. Leggiamo la terza ed ultima quartina di questo famoso componimento:

La vita che dà barlumi

è quella che sola tu scorgi.

A lei ti sporgi da questa

finestra che non sillumina.

E evidente che per Montale del decennio 1929-'39 la vita, pur nella sua problematica oscurità, è solcata da una serie di "barlumi", o, se si vuole, di "occasioni", che di tanto in tanto rompono il contesto duro e drammatico in cui la stessa sembra fisicamente e metafisicamente inchiodata. E possono essere "barlumi" o "occasioni" da poco, almeno di nessun valore apparente, oggetti o avvenimenti minimi, che ci possono permettere di sovrastare "i ciechi tempi", e cosí salvarci, nonostante tutto. A Liuba che parte, costretta dalle leggi razziali ad abbandonare la sua casa e la sua città, basta un nonnulla per il suo riscatto:

Non il grillo ma il gatto

del focolare

or ti consiglia, splendido

lare della dispersa tua famiglia.

La casa che tu rechi

con te ravvolta, gabbia o cappelliera?,

sovrasta i ciechi tempi come il flutto

arca leggera - e basta al tuo riscatto.

E a Dora Markus basterà a darle la forza per resistere persino un "amuleto", un topo bianco d'avorio che tiene "vicino alla matita delle labbra":

Non so come stremata tu resisti

in questo lago

d'indifferenza ch'è il tuo cuore; forse 
ti salva un amuleto che tu tieni

vicino alla matita delle labbra,

al piumino, alla lima: un topo bianco,

d'avorio; e cosí esisti.

Sono versi tratti da Dora Markus I, una poesia composta nel 1926, ma non a caso inclusa ne Le occasioni. Ed è l'attaccamento a queste cose umili e privatissime che solo forse può salvare l'uomo in tempi feroci, come quelli moderni, quando la storia è piena di "errori imperturbati", che nessun passaggio di spugna può per altro cancellare. Si legga la seconda strofe di Dora Markus 2, che risale al 1939:

\author{
La sera che si protende \\ sull'umida conca non porta \\ col palpito dei motori \\ che gemiti d'oche e un interno \\ di nivee maioliche dice \\ allo specchio annerito che ti vide \\ diversa una storia di errori \\ imperturbati e la incide \\ dove la spugna non giunge.
}

Non ci soffermeremo ancora a lungo su questo secondo libro della poesia montaliana. Diremo soltanto che nella cupa tragedia che pareva incombere sull'umanità in quegli anni, sino al punto che sembrava travolgerla e soffocarla, il poeta trova la forza per resistere a tanto scempio non mai rifugiandosi in facili illusioni, ma se mai aggrappandosi alla sua visione del mondo di cristiano laico che lo porta da un lato a respingere il "vano farnetico" e 1" amore di donne barbute", cioè delle monache che non credono che tutta la vita si viva su questa terra e che tutto l'amore si dia e si riceva in questo mondo, e dall'altro a liberarsi dalla disperazione, mostrandosi umanamente pietoso e insieme cosciente che "follia di morte non si placa a poco/prezzo. ". A resistere lo aiutano inoltre la fiducia negli affetti privati, specialmente l'amore della donna e per la donna, e la fiducia nella forza dell' umana dignità che ricorre a tutti i sortilegi possibili per sfuggire, appunto, alla "follia di morte"

Si giunge cosí all'esperienza de La bufera $e$ altro, un libro che contiene poesie scritte tra il 1940 e il 1954. Diciamo subito che si tratta di un libro molto composito, e se si pensa al tempo in cui fu composto non poteva forse essere diversamente. Tra il 1940 e il 1955 si chiusero e si aprirono problemi d'immensa portata per l'umanità, le conseguenze dei quali le stiamo vivendo noi oggi tra un'agonia e una speranza, che sembrano, ahimé, non volerci mai abbando- 
nare, e un poeta della forza morale e delle qualità intellettuali di Montale non poteva evidentemente non risentirne.

Se si esaminano le poesie di Finisterre, che sono ristampate nella prima sezione de La bufera, poesie tutte scritte tra il ' 40 e il ' 42 , e pubblicate nel " 43 a Lugano, è f́acile osservare che tematicamente e stilisticamente sono molto vicine a quelle della IV sezione, cioè l'ultima, de Le occasioni. C'è forse una tensione sentimentale e stilistica piú accentuata, specialmente nella prima che dà il titolo a tutto il libro, La bufera, appunto, e che simboleggia lo scatenarsi della guerra e i sogni e le speranze di pace e di cultura che non vogliono abbandonare l'umanità. Ma ci sono dei componimenti di eccezionale rigore espressivo e contenúísíico. Si pensi, per esempio, a Gli orecchini, una delle poesie piú concentrate e chiuse che Montale abbia mai scritto. La guerra, insomma, non fece perdere al poeta la compostezza, né sentimentale né súilistica, ma se mai gli permise d'interiorizzare ancora piú di prima l'enigma del significato del mondo e del supplizio a cui gli uomini sono condannati in momenti in cui nessun messaggio di pace e di speranza sembra che possa raggiungere il loro cuore e la loro inielligenza. Bellissima e assai significativa è a questo proposito la lirica Su tina letleza non scrilta che ora si può leggere, appunto, nella sezione Finisierie:
Per un formicolio d'albe, per pochi
fili su cui s'impigli
il fiocco della vita e s'incollani
in o:e $e$ in anni, oggi $i$ delfini a coppie
capriolano coi figli? Oh ch'io non oda
nulla di te, ch'io fugga dal bagliore
dei tuoi cigli. Ben altro è sulla terra.
Sparir non so né riaffacciarmi; tarda
la fucina vermiglia
della notte, la sera si fa lunga,
la preghiera è supplizio e non ancora
tra le rocce che sorgono t'è giunta
la bottiglia dal mare. L'onda, vuota,
si rompe sulla punta, a Finisterre.

La guerra con le sue violenze, con le sue distruzioni, con i suoi orrori morali e materiali, specialmente se si pensa che era una guerra assurda e insensata, trovava piú che sgomento, ansioso di sapere il poeta. Ma la violenza, specialmente la violenza assurda e irrazionale, la violenza mitica o mistica, che pure si annoda nel cuore della storia di sempre, e in quello della storia contemporanea forse in special modo, può mai essere capita e spiegata dalla ragione, almeno. dalla ragione 
come Montale la intendeva? Certamente no. Ecco perché nessuna bottiglia poteva arrivare dal mare e l'onda si rompeva, vuota, sulla punta, a Finisterre. Ma la ragione, e se proprio si vuole diremo la ragione borghese, anzi alto-borghese, come è sicuramente quella del nostro poeta, se la ragione si può mai qualificare veramente come aristocratica o borghese o proletaria, è stato lo scoglio a cui egli ha amato rimanere sempre attaccato durante la guerra e dopo la guerra. E questo forse è stato il punto massimo di forza e insieme il limite di Montale. La sua, infatti, è stata una ragione assoluta e non una ragione storica. E se questo gli ha permesso da un lato di scoprire e denunciare con forza chiaroveggente la fallacia di tanti miti che hanno ingannato ed intossicato l'uomo contemporaneo, ha pure contribuito dall'altro a bloccarlo di fronte alla storia, a impedirgli cioè di leggere la stessa in modo discriminante. Di qui il fatto che a un certo punto doveva necesariamente sfociare a una repulsione, quasi alla nausea della storia stessa. Quando la ragione, infantti, si arrocca in se stessa e si fa assoluta non può non sentire fastidio di tutte le scorie che la storia di necessità si porta dietro. Diciamo di necesità perché la storia non è e non può essere mai pura. Perciò se la ragione assoluta ci può aiutare a diagnosticare senza inganni e senza illusioni di alcun genere i mali della storia, e di questo l'umanità contemporanea molto deve essere debitrice a un poeta come Montale, è solo l'adesione alla ragione storica che ci può vietare d'isolarci aristocraticamente e può impedirci di sentire la nausea di tuffarci nel male che travaglia il mondo per cercare di espellerlo o almeno di limitarlo.

Per capire la tempra della razionalità alto-borghese e della moralità laico-cristiana con cui Montale si poneva di fronte alla realtà nel turbinoso tempo che sta tra la guerra e il dopoguerra, ci piace intanto ricordare le parole che concludono la Visita a Fadin. Sono parole di eccezionale importanza, oltre che molto chiare, tanto che si commentano da sole. Leggiamole: "Del colloquio non ricordo piú nulla. Certo non aveva bisogno di richiamarsi alle questioni supreme, agli universali, chi era sempre vissuto in modo umano, cioè semplice e in silenzio. Exit Fadin. E ora dire che non ci sei piú è dire solo che sei entrato in un ordine diverso, per quanto quello in cui ci muoviamo noi ritardatari, cosí pazzesco com'è, sembri alla nostra ragione l'unico in cui la divinità può svolgere i propri attributi, riconoscersi e saggiarsi nei limiti di un assunto di cui ignoriamo il significato. (Anch'essa, dunque, avrebbe bisogno di noi? Se è una bestemmia, ahimé, non è neppure la nostra peggiore.)

Essere sempre tra i primi e sapere, ecco ciò che conta, anche se il perché della rappresentazione ci sfugge. Chi ha avuto da te quest'alta lezione di decenza quotidiana (la piú difficile delle virtú) può attendere senza fretta il libro delle tue reliquie" 
E evidente che Montale non cercava, allora, come del resto non ha mai cercato, soluzioni extra storiche ai mali del mondo. Ma cercava delle soluzioni storiche, anzi riteneva che fossero possibili delle soluzioni storiche? Insomma, che cosa era possibile fare con la ragione, col sapere, e sia pure col sapere messo in corsivo? Soltando conoscere l'ordine pazzesco in cui si muove l'uomo nel mondo e al massimo esercitare la piú difficile delle virtú, la decenza quotidiana? In realtà, piú di questo gli pareva che non si potesse fare e andare al di là di questo pensava che fosse un inganno o un'illusione. L'intelligenza, dunque, non si doveva lasciar fuorviare da miraggi di sorta e doveva stare attentissima solamente a captare i segni della realtà, con la coscienza che il significato della stessa ci sfugge. Insomma, l'uomo al massimo poteva opporre ai mali del mondo, che l'intelligenza con sagacia e perspicacia doveva sapere indagare ed indicare, il rifiuto della coscienza morale. Ma andare oltre non si poteva. Pensare che la storia si possa in qualche modo emendare era, secondo il poeta, piú che un'illusione quasi un inganno morale che gli uomini tessono a se stessi.

Si capisce ora perché l'undici settembre del 1943 poteva scrivere il primo madrigale fiorentino e l'undici agosto del 1944 il secondo. Nel primo diceva:

Suggella, Herma, con nastri e ceralacca

la speranza che vana si svela, appena schiusa ai tuoi mattini.

Sul muro dove si leggeva Morte

A Baffo Buco passano una mano

di biacca. Un vagabondo di lassú

scioglie manifestini sulla corte

annuvolata. E il rombo si allontana.

E nel secondo:

Un Bedlington s'affaccia, pecorella azzurra, al tremolio di quei tronconi

- Trinity Bridge- nell'acqua. Se s'infognano

come topi di chiavica i padroni

d'ieri (di sempre?), i colpi che martellano

le tue tempie fin lí, nella corsia

del paradiso, sono il gong che ancora

ti rivuole fra noi, sorella mia.

Non c'è bisogno di osservare che col primo madrigale il poeta ribadiva, e, come sempre, con alta concentrazione poetica che qui si connota di un linguaggio beffardamente irriverente, com'è costume del popolo fiorentino nei suoi momenti piú caustici e irosi, la sua concezione 
della storia che non conosce che speranze vane; e nel secondo c'è il richiamo ai valori morali che sono i soli che ci posso salvare. Ma è nella Ballata scritta in una clinica, una poesia non tanto forse a torto privilegiata dal Contini, che l'orrore per la storia e la sofferenza della coscienza morale di fronte alla stessa, trovano un'espressione cosí intensa che rasenta l'agitazione. Ed è chiaro che l'autore si stava rinnovando anche nel linguaggio poetico. Vogliamo dire che non gli bastava piú il linguaggio ermeticamente chiuso del decennio precedente. La realtà ora comincia a prorompere a volte violenta e a volte irridente, ma anche nelle sue espressioni piú anonime e comuni. Insomma, il poeta tra la guerra e il dopoguerra tentò un nuovo scavo del reale, uno scavo piú aperto e sanguigno ma non meno complesso, anzi di una complessità ancora maggiore, se è vero, come a noi pare, che la sua indagine poetica sulla condizione dell'uomo contemporaneo raggiunge l'apogeo proprio in questo periodo.

A dimostrazione di quanto diciamo, si veda la sezione Silvae, che fu composta, come l'autore ci fa sapere in una nota, tra il ' 44 e il ' 50 e le Conclusioni provvisorie, l'ultima sezione de La bufera, che fu composta tra il ' 50 e il '54. Delle poesie della sezione Silvae ci soffermeremo sulle ultime due, vale a dire su $I l$ gallo cedrone e su L'anguilla, e specialmente su quest'ultima, che è, a nostro giudizio, la poesia piú bella, in senso assoluto, che Montale abbia mai scritto. Ma non va trascurata naturalmente neppure Voce giunta con le folaghe.

Ne $I l$ gallo cedrone ciò che colpisce è quel senso di acre realtà di dolore e di morte che il povero e bellissimo votatile, raggiunto dal breve sparo, sente. Ma non è solo l'animale che sente l'acre strazio del dolore e della morte, bensí anche il poeta. Il dolore e la morte dell' uccello, infatti, è anche il dolore e la morte del poeta. Ed è questo che fa grande e nuova questa poesia. Per Montale il dolore e la morte non sono scene soltanto da descrivere, come volevano $i$ veristi, o spettacoli solamente da godere, come volevano i decadenti, ma strazi invece da sentire nella propria carne, proprio come voleva il Leopardi. Non per nulla, e sia detto di passaggio, si può dire che Montale è il piú grande poeta moderno italiano dopo Leopardi. Certo, si sente anche in questa poesia che per il poeta genovese l'esperienza decadente non è passata invano, ma senza dubbio ha saputo trarsene fuori per giungere a un nuovo e piú vivo contatto con la realtà, anzi per sentire l'arsura e il bruciore vero della realtà, per raggiungere e cogliere cioè la realtà non piú solamente attraverso il simbolo, secondo la via maestra dei decadenti, ma attraverso tutte le sue manifestazioni tragiche e terribili, riprendendo cosí la strada che aveva battuto con successo il Leopardi. Un'altra osservazione che ci pare di poter fare ancora su questa grande poesia è circa la pietà che il poeta sente per l'infelice animale. Non si tratta di un momento di 
commozione sentimentale, come potrebbe essere quella di una signora bene educata e ben pasciuta, avvolta in qualche morbida pelliccia $o$ sprofondata in qualche comoda poltrona, che piange per la disgraziata sorte di una povera e bella creatura, bensí di un sentimento di pietà accorato, profondamente cristiano e leopardiano, scaturente dalla coscienza e dalla volontà che vorremmo tutti sottrarci al dolore e alla morte, ma non possiamo. Si leggano per quest'aspetto soprattutto la seconda e la terza quartina:

Chiede aiuto il singulto. Era piú dolce vivere che affondare in questo magma, piú facile disfarsi al vento che qui sul limo, incrostati sulla fiamma.

Sento nel petto la tua piaga, sotto un grumo d'ala; il mio pesante volo tenta un muro e di noi solo rimane qualche piuma sullilice brinata.

E nell'ultima quartina, dopo un richiamo a tutte le gioie e a tutti i giuochi della vita, gli occhi del poeta finiscono con l'appuntarsi sull'oscura e amara sorte a cui siamo fatalmente inchiodati.

Con L'anguilla, che per virtú tecnica e per altezza d'ispirazione è il componimento poetico di Montale che maggiormente si avvicina a La ginestra di Giacomo Leopardi, ci troviamo di fronte a una grandissima poesia. Si tratta di un componimento di trenta versi, tra versi lunghi, versi meno lunghi e versi brevi e brevissimi, che si snodano, anzi si torcono e si contorcono, in un solo periodo sapientemente modulato. Una prova di virtú tecnica eccezionale. Ma ovviamente non si tratta solamente di una manifestazione di virtú tecnica, che in poesia vale quello che vale, bensí del raggiungimento di una perfetta forma poetica che fa una cosa sola con la concezione della vita del poeta che è la fonte da cui egli trae ispirazione per la sua poesia.

L'anguilla incarna il simbolo della volontà e del desiderio di vita che è proprio di tutta la natura, di quella volontà e di quel desiderio di vita che non si ferma di fronte a nessun ostacolo e che cerca con tutte le forze in suo possesso di rompere e superare ciò che gli rende difficile la vita stessa. Gli sforzi che essa fa per trovare una sede adatta alla sua sopravvivenza, le difficoltà inaudite che deve superare per vincere "l'arsura e la desolazione", riuscendo a trovare paradisi d'amore e di fecondazione nei posti piú impensati e impossibili, il suo tendersi e contorcersi tra i macigni, il suo infiltrarsi "tra gorielli di melma" per riemergere tra "pozze d'acqua morta", è ciò che 
la rende sorella all'uomo. L'uomo dunque, come l'anguilla, si dibatte e guizza tra mille difficoltà e la via che percorre per poter vivere non è mai sgombra di ostacoli di ogni genere. Non in modo facile e piano perciò può realizzarsi la nostra esistenza, anche se bisogna aver sempre fede che tutto comincia quando tutto pare / incarbonirsi, bronco seppellito.

Ma è nelle Conclusioni provvisorie, cioè nel Piccolo testamento e ne Il sogno del prigioniero, che possiamo trovare riassunta in modo chiarissimo ed incisivo la sua posizione ideale e morale del tempo degli anni del dopoguerra che sfociarono nella guerra fredda. Queste poesie, infatti, furono scritte tra il ' 50 e il ' 54 . In un mondo ideologicamente diviso in modo verticale e quasi manicheamente contrapposto, con le forze conservatrici all'attacco in tutta l'Europa e in tutto il mondo e con le forze proletarie e rivoluzionarie bloccate dai dogmi e dal mito di Stalin, in un mondo dove pareva soffocato ogni respiro di libertà reale e al di fuori dei grandi partiti che irrigimentavano le masse non pareva che ci potesse essere possibilità di movimento, il poeta manifestava la sua fede non alimentata di lumi di chiesa o di officina che confortassero "chierico rosso, o nero" $\mathrm{E}$ evidente che la polemica coi democristiani e coi comunisti qui diventa aperta. E non c'è bisogno di vedere necessariamente nella sardana infernale dell'ombroso Lucifero che sta per scendere su una prora del Tamigi, dell'Hudson, della Senna/scuotendo l'ali di bitume semimozze dalla fatica. ., come pure è stata vista, un'allusione alla paura, che era proprio della borghesia di quegli anni, che l'Europa occidentale potesse cadere preda dell'armata rossa. La verità è che per Montale le speranze di rinnovamento e di progresso che gli anni del dopoguerra avevano fatto sorgere nel cuore di masse sterminate non erano altro che illusioni, tanto piú ingannevoli quanto piú accettate senza distacco e ragione critica. Perciò alle illusioni che seguivano le masse, opponeva orgoglisamente la propria concezione del mondo smagata e amara, l'unica che non permetteva disinganni e che non fosse un fuoco di paglia, tanto che diceva:

Giusto era il segno: chi l'ha ravvisato
non può fallire nel ritrovarti.
Ognuno riconosce i suoi: l'orgoglio
non era fuga, l'umiltà non era
vile, il tenue bagliore strofinato
laggiú non era quello di un fiammifero.

E ne Il sogno del prigioniero ribadeva la sua sfiducia nella possibilità che il mondo potesse cambiare in meglio. Lo vedeva minato quasi da un male metafisico, a tal punto che diceva: 
La purga dura da sempre, senza un perché.

Dicono che chi abiura e sottoscrive

può salvarsi da questo sterminio d'oche;

che chi obiurga se stesso, ma tradisce

e vende carne d'altri, afferra il mestolo

anzi che terminare nel paté

destinato agl'Iddii pestilenziali.

E chiaro che il poeta vedeva con chiarezza il nuovo conformismo morale e ideale ammorbante che si stava abbattendo sull'Europa del dopoguerra, spazzando via gl'ideali e le aspirazioni della Resistenza proprio in quegli anni. Si ricordi che si stava ritornando, in forme piú subdole e ipocrite, alla necessità di avere una certa tessera politica per ottenere un posto o far carriera. L'aria politica e morale stava diventando cosí nuovamente irrespirabile e sembrava pesare come una cappa di piombo. Perciò non ci poteva essere riposo e pace per gli uomini. E il prigioniero non poteva non concludere il suo discorso col richiamo a un sogno di speranza e di felicità che il cammino della storia col suo assurdo dispiegarsi sembrava negare. Si ponga mente agli ultimi quattro versi con cui si chiude il componimento:
e $\mathrm{i}$ colpi si ripetono ed $\mathrm{i}$ passi,
e ancora ignoro se sarò al festino
farcitore o farcito. L'attesa è lunga,
il mio sogno di te non è finito.

Ma non dovevano passare molti anni ancora perché il sogno finisse e perché il prigioniero facesse definitivamente $\mathrm{i}$ conti in negativo con la storia. Di questo nuovo momento della poesia montaliana sarà frutto Satura, il libro che raccoglie la produzione poetica del poeta genovese tra gli anni 1962-'70.

Si badi subito, intanto, agli anni in cui fu composto il libro. E noto che fu quello il tempo in cui il fenomeno piú grosso e appariscente del dopoguerra sembrò raggiungere i risultati piú cospicui e nello stesso tempo rivelò la sua crisi irriversibile. Alludiamo al fenomeno del nascere e dell'affermarsi del neocapitalismo con tutto ciò che esso rappresentò per tutta l'Europa sia sul piano delle strutture che su quello delle sovrastrutture, fenomeno che raggiunse, appunto, il suo apogeo tra gli anni '60-' 65 , mentre nel ' 68 , l'anno delle lotte studentesche che investirono tutte le grandi Università europee, da Roma a Berlino, da Parigi a Pisa, rivelò che il neocapitalismo non solo era in crisi, ma che soprattutto non aveva risolto nessun problema vero di quelli che si erano affacciati alla coscienza europea subito dopo la fine della guerra, nonostante le tante illusioni che aveva creato intorno agli anni ' 60 . Si rifletta un momento su 
ciò che significò il fenomeno neocapitalistico per tutta l'Europa occidentale, e specialmente per un paese come l'Italia che non aveva mai veramente avuto nel passato una vera e grande borghesia indutriale. Significò innanzi tutto il passaggio rapido e caotico da una economia prevalentemente agricola a una economia industriale col conseguente spostamento di masse imponenti dalla campagna alla città. Fu in quegli anni, infatti, che il fenomeno dell'emigrazione interna ed esterna in Italia riprese con vigore. Milioni e milioni di persone lasciarono il mezzogiorno e le isole e si trasferirono al nord, al nord della penisola e al nord dell'Europa. La classe dirigente pensò cosí di poter rispondere all'ansia di progresso delle masse e di poter risolvere in questo modo $\mathrm{i}$ problemi delle stesse, sia quelli di ordine materiale che di ordine morale. In realtà non fece altro che crearne altri e piú gravi. E in primo luogo si dette origine al fatto piú vistoso che il neocapitalismo portava inevitabilmente con sé, vale a dire al dato dell' assalto incontrollato e incontrollabile ai beni della natura e all'anima della gente che veniva trasformata in un robot non pensante $\mathrm{e}$ in una perfetta macchina di consumo. Si ricordi che fu proprio in quegli anni che si coniò ed ebbe fortuna il termine consumismo.

Quanto ai fenomeni sovrastrutturali a cui il neocapitalismo dette origine ricorderemo soltanto il neopositivismo logico ed epistimologico in filosofia, lo strutturalismo nel campo della linguistica e della critica letteraria, le nuove avanguardie letterarie che fiorirono un pò in tutta l'Europa, compresa naturalmente l'Italia, tutti fenomeni che si connotavano per la loro modernità e scientificità come aideologici, mentre i loro propugnatori e seguaci non si accorgevano, o non se ne volevano accorgere, che l'aideologia era ciò che ci voleva in massimo grado perchè il neocapitalismo si affermasse incontrastato e mercificasse o meglio merdificasse, come dicevano icasticamente e con spregio coloro che non si vollero far complici delle sue fortune, lo spirito.

Vediamo ora quali ripercussioni questi anni e questi avvenimenti ebbero su di una personalità come quella di Montale. Comincieremo col dire, intanto, che questi anni lo trovano collaboratore, in qualità di giornalista stipendiato, de "Il corriere della sera", il piú grande giornale borghese italiano e uno dei piú grandi e famosi giornali borghesi di tutta l'Europa. Frutto della collaborazione a "Il corriere della sera" furono gli scritti che poi saranno raccolti nei due volumi Auto da fé e La farfalla di Dinard. La collaborazione del nostro poeta a "Il corriere della sera" è stata, come è noto, variamente interpretata e commentata. Per quanto ci riguarda diremo che certamente ebbe un preciso significato per il poeta e per il giornale: per il poeta che potette cosí far giungere la sua voce amara e disillusa ad un pubblico 
vasto e per il giornale che potette vantarsi non soltanto di avere tra i suoi collaboratori un poeta celebre, ma anche perché attraverso di lui potette mostrare con quale animo la grande borghesia, di cui 'Il corriere della sera" era voce ed espressione, si acconciò ad accettare i fasti e i nefasti del neocapitalismo. Perciò a noi pare per lo meno schematica, oltre che semplicistica, la tesi di coloro che hanno voluto vedere nella collaborazione di Montale a "Il corriere della sera" la naturale adesione di un grande borghese, in senso ideale, ad uno dei piú grandi giornali della borghesia europea. La verità è piú complessa, e secondo noi va cercata proprio nell'atteggiamento, niente affatto entusiasta, che la grande borghesia ebbe nei confronti del neocapitalismo e delle forze politiche che lo appoggiarono. Sicché l'incontro tra il poeta che guardava con occhi per niente compiaciuti all'affermazione neocapitalistica e il giornale che rispecchiava gl'interessi della grande borghesia italiana, ha un significato ideale ben preciso. Fu si, infatti, un incontro ideologico, ma non nel senso che Montale aderiva all'ideologia borghese neocapitalistica, che fu tra l'altro superficialmente ottimistica, oltre che pacchiana e incolta, ma se mai nel senso che guardava con forti riserve, sino alla repulsione, proprio come faceva la grande borghesia, o almeno parte di essa, alle fortune del neocapilismo.

Se si vuole ora capire con quale animo il poeta guardava ai risultati che si stavano raggiungendo in Italia intorno agli anni ' 60 , gli anni per intenderci del cosí detto boom economico e quando pareva che per il popolo italiano stessero per aprirsi le porte dell'Eldorado, si veda Botta e risposta I, che fu composta nel 1961 e che è la poesia con cui si apre Satura. Si tratta di un componimento eccezionale per la forza dissacrante che contiene in sé. Viene tracciato un breve profilo morale della storia italiana a cominciare dal tempo dell'adolescenza dell'autore, per terminare, appunto, agli anni ' 60 . Ebbene, ciò che s'impone alla nostra attenzione è innanzi tutto l'atmosfera irrespirabile e nauseabonda che circola in tutta la poesia. Il linguaggio piú che realistico, si può considerare saturo di fetori rivoltanti. Si rifletta alle varie espressioni che si susseguono nel corso del componimento. Il poeta rievocando i tempi del fascismo di altro non si ricorda se non di corridoi "sempre piú folti di letame", dove "si camminava male e il respiro mancava" e dove crescevano di giorno in giorno i "muggiti umani" Non manca poi un accenno alla condizione morale a cui si erano ridotte a vivere le masse, che sono viste come se si nutrissero di "un'infilzata fetida di saltimbocca" Sicché in quell'aria irrespirabile, fu possibile sopravvivere soltanto grazie a qualche barlume che Gerti o Liuba o Dora o Clizia o la Mosca o la Volpe, le varie donne-angelo del poeta insomma, seppero di tanto in tanto emanare, ispirando coraggio e speranza in tanta miseria. Ma come si presentano le cose nel dopoguerra, dopo "il tonfo: l'incredibile"? Ascoltiamo il poeta: 


\begin{abstract}
A liberarci, a chiudere gli intricati cunicoli in un lago, bastò un attimo allo stravolto Alfeo. Chi l'attendeva ormai? che senso aveva quella nuova palta? e il respirare altre ed eguali zaffate? e il vorticare sopra zattere di sterco? ed era sole quella sudicia esca di scolaticcio sui fumaioli, erano uomini forse, veri uomini vivi

i formiconi degli approdi?
\end{abstract}

Le cose, come si vede, non cambiano per nulla nel dopoguerra, tanto che solo una "nuova palta" si aggiunge all'antica e si continua a vorticare sopra "zattere di sterco" Si pensi ancora ai termini che l'autore ama usare in questa poesia e si vedrà che non è certo avaro di parole come letame, infilzata di saltimbocca, bastioni fecali, bargelli del brago, nuova palta, altre ed eguali zaffate, zattere di sterco, sudicia esca di scolaticcio sui fumaioli, formiconi degli approdi, non può nascere l'aquila dal topo, quasi a voler sottolineare il marciume ideale e morale a cui si è ridotto o è stato ridotto l'uomo del ' 900 , il quale non conosce altra possibilità di vita se non quella di una condizione fecale. Mai Montale era giunto a tal punto di repulsione nei confronti della storia contemporanea. Si tratta quasi di una repulsione fisica, oltre che morale.

Come spiegarsi ora tutto questo se non riflettendo a fondo su quanto avvenne in Europa, e specialmente in Italia, nel famoso decennio che grosso modo va dal ' 55 al ' 65 , il decennio del boom economico, della diffusione della televisione e dell'accettazione da parte delle masse indifese della filosofia del consumismo imposta dal neocapitalismo e quando gli ideali e le speranze della Resistenza furono colpiti, o per lo meno si tentò di colpirli, nel cuore e quando "i formiconi degli approdi" vale a dire certi gruppi sociali e certe persone che il poeta si rifiuta di considerare "uomini, uomini vivi", e che sembravano essersi eclissati come "topi di chiavica", per dirla sempre con lo stesso poeta, al tempo della Resistenza, ritornarono invece di nuovo in auge? $\mathrm{E}$ fu proprio allora, quando cioè sembrò afflosciarsi nuovamente la tempra morale e ideale del popolo italiano, quando il conformismo si contrabbandò per scienza del saper vivere, quando i superficiali brillanti senza alcuna fede furono preferiti agli uomini di credenze sincere e sofferte, quando inoltre ancora una volta le virtú oscure di tutti gli uomini semplici sembrarono destinate ad essere sacrificate, che Montale giunse a maturare il suo distacco definitivo dai miti della storia e a considerare la resistenza interna l'unica trincea inespugnabile dell' umana dignità. Perciò ai tronfi messaggi e 
alla stolte illusioni, agli atteggiamenti degli eroi e dei martiri antepose l'oscura attività di chi non si aspetta nulla dal mondo perchè sa che è solo una pretesa quella di coloro che credono che esistere su questa terra "sia veramente possibile", che credono cioè che l'avvento di un ordine nuovo possa curare i mali del mondo. Si veda per questa conclusione Botta e risposta III, che risale al '68:

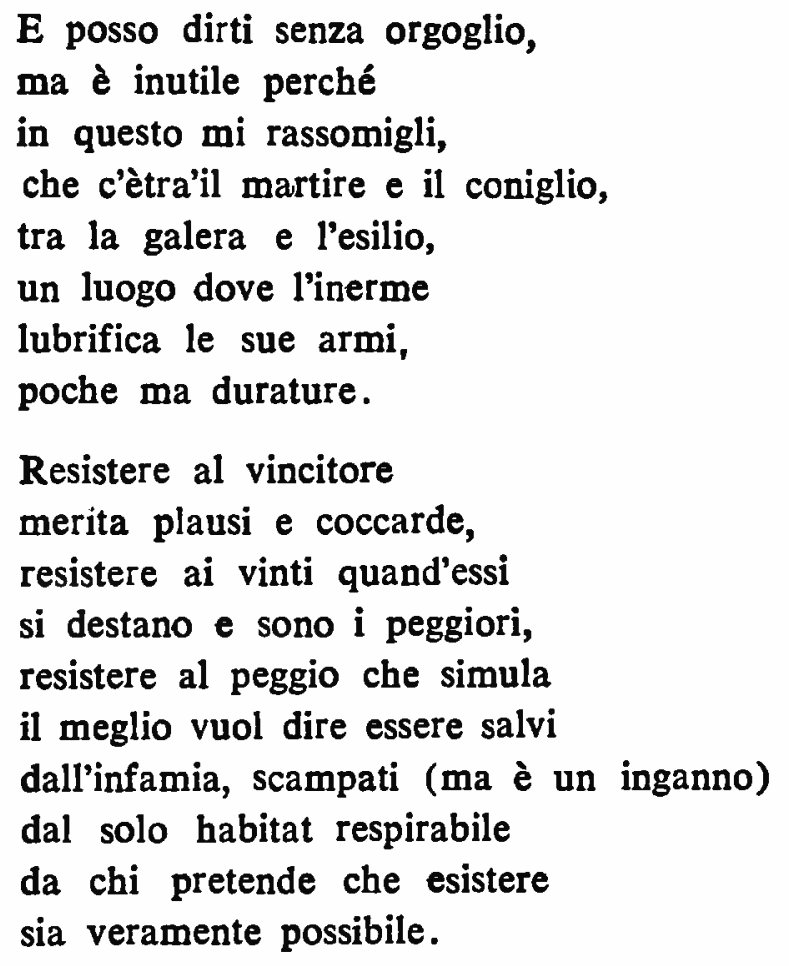

Si capisce ora perché nel ' 69 potette comporre le due famose liriche intitolate $\mathrm{La}$ storia, oltre quella intitolata Fanfara. Occorre soffermarsi un momento su queste liriche, che possiamo considerare come il punto d'arrivo della riflessione del nostro poeta su l'essere e il destino del mondo contemporaneo, una riflessione cominciata certamente in tempi lontani, come abbiamo cercato di dimostrare in questo studio, ma conclusa non a caso intorno agli anni ' 70 , vale a dire quando l'assetto storico nazionale e internazionale che era scaturito dalle vicende che si erano susseguite alla seconda guerra mondiale, sembrò cadere in una crisi di cui noi oggi stiamo vivendo i sussulti piú o meno violenti. E non paia strano che le conseguenze di tale crisi, il cui punto di partenza manifesto si può far risalire al 1968, e che fu una crisi che investí tutta l'Europa, si stiano ripercuotendo particolarmente su l'Italia. La ragione va cercata nel fatto che in Italia, che si presenta come un paese culturalmente assai vivo e avanzatissimo e insieme economicamente piuttosto debole, almeno rispetto ad altri paesi capitalisticamente assai sviluppati, si sta tentando di percorrere vie nuove ed originalissime per risolvere quella massa ingente di problemi, e sono problemi di libertà e di giustizia, di avan- 
zamento civile insomma, che non furono risolti dopo la seconda guerra mondiale, che anzi non furono nemmeno programmaticamente impostati, e che oggi travagliano la coscienza non solo dell'Europa, ma di tutto il mondo. E si sa che quando si percorrono vie nuove il cammino non è mai né sicuro né spedito. Perciò non c'è da maravigliarsi se l'Italia di oggi si presenta con il volto di un'officina sperimentale, con tutto ciò che un fatto del genere significa.

Ma ritorniamo alle liriche La storia e Fanfara del nostro autore, che, come abbiamo detto sopra, risalgono al ' 69 e che possono essere considerate il punto d'arrivo della sua riflessione sull'essere e il destino dell'uomo contemporaneo. Come vede Montale in queste poesie la realtà della storia? Certamente non come uno sgranarsi di avvenimenti che si possono spiegare con la ragione o che possono essere giustificati dalla stessa. Ed è questo, senza dubbio, che lo distingue sia dagli idealisti che dai materialisti, come d'altra parte dai cattolici e dagli spiritualisti in generale. La storia per lui non è idealisticamente un prodotto dello spirito e della ragione cosí come non è materialisticamente un portato piú o meno necessario delle strutture economiche di una determinata società, e tanto meno è un fenomeno che si può spiegare o giustificare provvidenzialisticamente. La storia perciò per il nostro poeta non ha una direzione, non ha un fine e non ha nemmeno una tendenza; non ha un prima e non ha un poi e quindi non ha uno svolgimento, e meno che mai un progresso. Sicché la concezione dello sviluppo dialettico della storia, sia di tipo idealistico che di tipo materialistico, è una pura invenzione dei filosofi e non corrisponde alla realtà, la quale non conosce nessuna direzione, nessuno sviluppo e nessun progresso. Che cosa è allora la storia per Montale? Si direbbe che per lui la storia non esiste proprio, in quanto esiste soltanto il fenomeno fisico e metafisico della vita che del resto non ha nessun senso o che per lo meno sfugge alla nostra ragione. Si capisce perciò anche perché per il nostro poeta non significa niente essere pessimisti o ottimisti. Per gli ottimisti il mondo è qualcosa di godibile e di perfettibile; per i pessimisti no. Gli uni e gli altri quindi credono a una realtà del mondo, sia pure di segno contrario. La realtà invece nell'intuizione montaliana è imprevedibile ed è senza direzione e senza senso e come tale non può essere né consolante né desolante. L'uomo dalla storia, come dalla vita, si deve aspettare tutto e il contrario di tutto. E se crede che il suo destino sia migliore o peggiore di quello toccato ad altri, s'inganna. Si rifletta a quanto il poeta dice nella seconda strofe della poesia La storia 2:

La storia gratta il fondo

come una rete a strascico

con qualche strappo e piú di un pesce sfugge.

Qualche volta s'incontia l'ectoplasma 
d'uno scampato e non sembra particolarmente felice.

Ignora di essere fuori, nessuno glie n'ha parlato.

Gli altri, nel sacco, si credono

piú liberi di lui.

Questa è la condizione umana, dunque: essere sí nella storia, ma non sapere che cosa questa sia, né dove porti, né quale destino ci riservi. $\mathrm{Si}$ veda a questo proposito anche Fanfara e si pensi a come il poeta irride alle bubbole/ che spacciano i papisti/ modernisti o frontisti/ popolar/ gl'impronti!

E chiaro a questo punto che Montale con Satura scopriva definitivamente le sue carte di grande borghese che ha voluto leggere nel cuore problematico dell'uomo contemporaneo con gli occhi non velati da alcuna illusione derivante specialmente dalla credenza piú o meno dogmatica che l'avvento di questa o quella ideologia politica o di questo o quel credo filosofico, morale o religioso, possa da solo modificare la realtà fisica e metafisica in cui gli uomini sono involti e possa indicare altresí da solo all'uomo la via della salvezza morale e della libertà e della dignità, ma con la coscienza invece che soltanto la ragione che non chiude gli occhi di fronte alla realtà, che si mostra sempre difficile e imprevedibile, ci può dare anche la forza di resistere alle storture di cui sono intessute sempre inevitabilmente la storia e la vita e ci può liberare inoltre dal pessimismo che non approda a nulla, che anzi si fa complice di quelle storture, senza farci cadere d'altra parte in un ottimismo d'obbligo che oltre che falso è anche fatuo. 